\title{
INFRAESTRUCTURA PARA EL DESARROLLO
}

\section{Iván Calero Hidalgo*}

El desarrollo de un país sin infraestructura física básica es impensable; por otra parte, la calidad, cobertura, seguridad y eficiencia de la infraestructura son réditos del desarrollo. La forma como sean enfrentados los retos que plantea esta interdependencia marca, en buena parte, el bienestar o estancamiento de los pueblos. Por
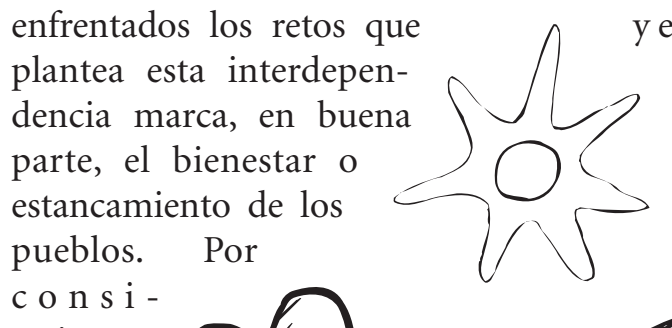

$\mathrm{t}$ e , e $n$

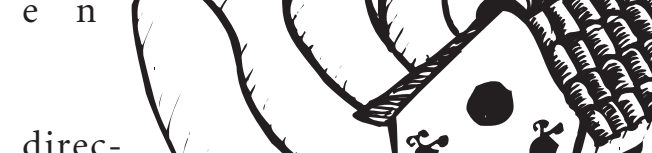
dirección al progreso, las soluciones a los problemas del desarrollo exigen, en relación a las políticas, solidez y continuidad, en tanto que, en esas políticas, eficiencia y eficacia, concordantes con el escenario económico vigente, caracterizado por sus limitados recursos financieros, frente a amplias y crecientes demandas. De otra parte, el crecimiento poblacional y el deterioro del entorno territorial y sus recursos naturales renovables, exigen racionalidad y alcance integral en dichas políticas y soluciones.

Bajo esta perspectiva y a partir de casos concretos, queremos compartir nuestras experiencias, comentarios y sugerencias, sobre los relación a los proyectos que plasmen

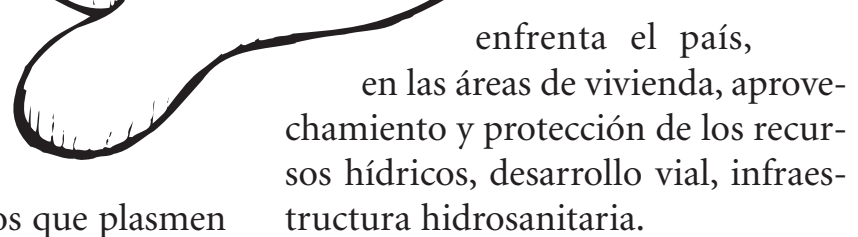

problemas de infraestructura básica que tructura hidrosanitaria.

\footnotetext{
* Director de la Carrera de Ingeniería Civil de la Universidad Politécnica Salesiana-Ecuador.
} 
En esta primera entrega, nos referiremos al problema de la vivienda de interés social, considerando dos modelos para resolverlo: el primero, cuestionable por los resultados e impactos que evidencia; el segundo, que involucra activa participación de la comunidad organizada $y$, por lo pronto, tiene una sola experiencia en Ecuador, de las pocas en América Latina. Respecto al primer modelo, nos limitaremos a consignar las conclusiones planteadas en una evaluación específica realizada sobre el programa de vivienda social más representativo, por el número de familias involucradas, ejecutado en nuestro país en los últimos diez años; sobre el segundo modelo, a partir de su aplicación en el Programa de Vivienda "Quitumbe", que se desarrolla en el Sur de Quito, la Asociación de Cooperativas Múltiples "Solidaridad", analizaremos sus particularidades y plantearemos sugerencias de acciones complementarias que, a nuestro criterio, irán en dirección a la consolidación del nuevo modelo. Finalmente, enfocaremos la participación de la universidad en los proyectos de desarrollo, a partir de dos elementos: 1) nuestra cooperación en el primer programa ecuatoriano de producción social de la vivienda; $y, 2)$ una importante ponencia, presentada en el Primer Congreso
Internacional "Universidad, Desarrollo y Cooperación", desarrollado del 25 al 27 de abril de 2007, en la ciudad de Cuenca.

\section{PARTE I. VIVIENDA Y DIG- NIDAD}

\section{Vivienda de interés social - un problema prioritario}

Para el ser humano la vivienda es el derecho vital más importante luego de la alimentación, además es el espacio íntimo de la familia como célula social y el ámbito de nuestra privacidad, inviolable en todos los estados democráticos del mundo. Por tanto, el derecho a la vivienda como necesidad vital, como espacio de desarrollo familiar, y ámbito de privacidad humana, debería estar garantizado en todas las latitudes del planeta. Esta garantía se refiere, principalmente, a la vivienda de interés social, entendida como el hábitat para familias que, por su nivel económico, no tienen acceso a la vivienda comercial y, por consiguiente, tampoco a crédito directo convencional del sector financiero. Además, esta garantía es coherente con los principios de racionalidad y equidad establecidos en varios foros internacionales especializados, como el X 
Foro Iberoamericano de Ministros de la Vivienda, donde bajo la premisa “... que, las políticas gubernamentales en materia de vivienda, deben tener carácter estructural continuado y permanente, y en cuya adopción se debe considerar una amplia participación de la ciudadanía y de los gobiernos locales..."1, se acordó, entre otras acciones, proponer a los gobiernos “... el establecimiento de fuentes de financiación (públicas, privadas e internacionales) suficientes, estables y continuadas para el sostenimiento de las políticas de vivienda..., bajo principios de equidad social"2.

Lamentablemente, en buena parte de los países en desarrollo, incluido el nuestro, el derecho a la "vivienda digna" no pasa de declaración, utilizada en la práctica como plataforma demagógica con fines electorales. Y, cuando algo se hace para cumplir las promesas de campaña, casi por norma, las acciones son fuente de lucro indebido, finalmente facturado a las víctimas del engaño, en tanto que los tugurios construidos como "soluciones habitacionales", imposibilitan el ejercicio, aún ele- mental, de la ciudadanía y, por tanto, atentan a la dignidad humana.

\section{Necesidad de nuevas políti- cas de vivienda}

La alta demanda de vivienda de interés social, la escasez de recursos para desarrollar programas que atiendan esa demanda y los resultados desalentadores obtenidos en nuestro país, salvo excepciones, evidencian la necesidad de elevar las políticas de vivienda, formulándolas como parte de una planificación coherente y desburocratizada, al margen de coyunturas y compromisos electorales, como lamentablemente se ha hecho costumbre en Ecuador. La racionalidad de las políticas no sólo permitirá enfrentar el problema de vivienda social con proyectos integrales que, a más de la autogestión involucren la participación de organismos seccionales y agentes sociales sin fines de lucro, sino que generará base y credibilidad, para la disponibilidad de recursos extrapresupuestarios dirigidos al sector. En esta dirección, de fundamental importancia es contar con

1 "Declaración de Guadalupe". X Foro Iberoamericano de Ministros del Sector de la Vivienda y Desarrollo Urbano. Guadalupe, septiembre de 2005.

2 (Ley de Consultoría. Codificación 24. Registro Oficial 455 de 5 de noviembre de 2004). 
organizaciones comunitarias que tengan claros objetivos de autogestión y transparencia de gestión, organizaciones desvinculadas de políti$c o s$, de salvadores o expertos en recetas burocráticas.

Ciertamente, como se evidenciará adelante, la comunidad organizada, con convicción y creatividad, pero también con transparencia, se ha adelantado al estado ecuatoriano, probablemente más de un decenio, en la generación, aplicación y validación de una propuesta integral para solucionar el problema del hábitat de interés social.

\section{Dos opciones para comparar}

En las actividad de vinculación con la colectividad, de la carrera de Ingeniería Civil de la Universidad Politécnica Salesiana, hemos tenido la oportunidad de conocer y evaluar las prácticas y resultados en dos opciones, diametralmente diferentes, para enfrentar el déficit de vivienda social. Por una parte, una forzada por el clientelismo político y el compromiso electoral: opción de paternalismo irresponsable, generador de problemas sociales, urbanísticos y ambientales, en muchos casos irreversibles, opción que, en gran medida, parecería desprecia la dignidad humana y la capacidad organizativa y productiva de la comunidad. Por otra parte, una opción de producción social de la vivienda: opción sustentada en la sinergia del derecho y el deber, del esfuerzo personal y la solidaridad, de la autogestión y la transparencia.

\subsection{Una opción con incertidumbre}

Por pedido a nuestra Universidad por parte de la Comisión de Defensa del Consumidor, del Usuario, del Productor y Contribuyente; del $\mathrm{H}$. Congreso Nacional, la carrera de Ingeniería Civil realizó, en el segundo semestre del año pasado, la evaluación de los programas de vivienda popular Primavera I y Primavera II, ejecutados por uno de los últimos gobiernos, en cumplimiento de lo que, en su oportunidad, fue uno de los principales ganchos de su propuesta electoral. Como parte de los dos programas han sido construidas 813 viviendas, para igual número de familias de escasos recursos económicos. Sobre la base del análisis técnico objetivo, nuestro informe de evaluación evidencia una realidad dolorosa para un grupo social vulnerable, en cuyo perjuicio parecería se han confabulado la inmoralidad y la negligencia. En los siguientes puntos se resume el contenido del informe. 


\subsubsection{Apreciación general}

a) Deficiencia generalizada del proceso constructivo;

b) Inobservancia de las normas mínimas del Código ecuatoriano de la construcción;

c) Mala calidad de los materiales de construcción.

\subsubsection{Conclusiones principales}

a) Las viviendas de los programas evaluados, no cumplen condiciones mínimas de habitabilidad;

b) La precariedad del hábitat de ninguna manera refleja el costo real, que incluye el monto de la deuda que ahora pesa sobre los "beneficiarios" y el valor del bono de la vivienda.

\subsection{Impacto social}

Los "beneficiarios" del programa, más de ochocientas familias, sólo conocieron las viviendas que habían soñado, cuando estas estuvieron construidas y luego de haber suscrito los documentos que aseguran el pago de las deudas adquiridas con el Estado por los tugurios. A continuación, el sueño, iniciado en una campaña electoral, se transformó en pesadilla. Probablemente la pesadilla continúa. No cabe duda que la situación humana de esas familias ha sufrido grave deterioro.

\subsection{Una opción diferente}

También gracias a nuestra actividad de vinculación con la comunidad, desde hace cinco años, la carrera de Ingeniería Civil mantiene relación de colaboración mutua, con la con la Asociación de Cooperativas Múltiples de Quito "Solidaridad". En este caso, el problema de la vivienda se resuelve desde una óptica diferente: utilizando un modelo de producción social del hábitat. Las características del modelo y la estructura organizativa aplicada hacen que esta experiencia sea única en el país y una de las pocas en América Latina. La actividad fundamental de nuestra carrera ha sido y es la fiscalización técnica.

\subsubsection{Producción social de la vivienda} en el ejemplo de "Solidaridad"

Un ejemplo, probablemente el más importante en nuestro país, sobre autogestión en la producción social de la vivienda, es el Programa de Vivienda Quitumbe que desarrolla en el Sur de Quito la Asociación de Cooperativas Múltiples "Solidaridad" (una organización con dieci- 
siete años de existencia, conformada por trece cooperativas y otras organizaciones sociales, agrupa a más de dos mil quinientas familias, ha creado varias microempresas con autogestión, vinculadas con las áreas de vivienda, educación, alimentación, salud, ahorro y crédito, turismo comunitario, formación y capacitación). De esta experiencia, es posible obtener importantes conclusiones respecto a organización y participación comunitaria; organización y administración de la construcción de vivienda con autogestión; educación, protección y desarrollo ambiental sostenible, solidaridad social, etc. De particular importancia son los elementos de este programa, vinculados con la autogestión, puesto que más allá de asegurar la viabilidad financiera del programa, han generado efectos sinérgicos de valor material, pero sobre todo de fe, autoestima, creatividad, solidaridad y compromiso con la protección sostenida del entorno ambiental. Sumemos a esto, la eliminación de las corruptelas, propias de la actividad estatal en los procesos de contratación, construcción y fiscalización. A los elementos de autogestión, que en Quitumbe han originado efectos de signo contrario a los que generan el falso paternalismo y el clientelismo demagógicos, probablemente debemos considerarlos como condiciones imprescindibles de los programas de vivienda social que se formulen en el país, con racionalidad y transparencia. Enumeremos algunos de dichos elementos:

a) La estructura organizativa cuenta con personería jurídica y como persona jurídica ha escriturado y registrado la propiedad sobre bienes inmuebles, asî como ha obtenido permisos municipales para urbanización y construcción de vivienda;

b) Se ha logrado diversificar las fuentes para la captación de recursos financieros, accediendo también al sector financiero convencional. Este es un signo de solidez y confiabilidad de la organización;

c) Se ha eliminado la informalidad en la tenencia de la tierra y las ilegalidades en su comercialización;

d) A través de las condiciones de autogestión, se ha descartado el ingreso al programa de vivienda de potenciales traficantes e intermediarios, mal común en los programas estatales de vivienda popular;

e) Se ha posibilitado la ejecución de un plan integral de convivencia humana, sin atentar al 
derecho de privacidad. El carácter integral se refiere a seguridad jurídica de tenencia, disponibilidad de servicios y equipamiento, habitabilidad, desarrollo comunitario participativo, solidario e incluyente, desarrollo urbano sustentable, protección del entorno territorial y sus recursos naturales;

f) Ha contado, durante la planificación y ejecución del programa con asistencia técnica de organismos seccionales y de la universidad. En efecto, el Municipio Metropolitano ha tenido activa participación en la etapa de planificación, en tanto que la Universidad Politécnica Salesiana lo ha hecho durante la ejecución del programa, tomando a su cargo la fiscalización técnica de la construcción;

g) A través de un liderazgo participativo se ha eliminado la explotación, manipulación y clientelismo;

h) Se ha posibilitado el desarrollo microempresarial en la comunidad para diferentes servicios, particularmente, los relacionados con fabricación de materiales de construcción y con procesos constructivos;

i) La autogestión participativa ha consolidado la equidad de gé- nero, posibilitando la intervención de la mujer, en la planificación, adopción de decisiones y ejecución de políticas y acciones. De hecho el carácter integral del programa sería inconcebible sin una real equidad de género;

j) Se ha elevado la conciencia ambiental y la autoestima de la comunidad vinculada;

k) Constituye una opción validada de cómo atender con racionalidad el derecho de las familias a una vivienda digna y de cómo, resolviendo el problema de la vivienda, contribuir al desarrollo urbano armónico y ambientalmente sostenible;

1) La autogestión ha asegurado el control de calidad de los materiales y procesos constructivos, pues en lo que tiene que ver con la protección a la vida y el respeto a la dignidad humana, no puede haber diferencia entre la vivienda que es de interés social y la que no lo sea. En este aspecto, "la relajación de normas de construcción demasiado restrictivas" a que, en uno de sus documentos, se refiere la Comisión sobre el Desarrollo Sostenible del Consejo Económico y Social de las Naciones Unidas, obviamente no signifi- 
ca "relajo" y "festín" para enfrentar los problemas relacionados con la vivienda de interés social, como parece han asumido algunos de nuestros "expertos" en interpretar, de acuerdo a su interés, constituciones, leyes, reglamentos, convenciones, etc.;

m) Las inversión realizada en el Programa de Vivienda Quitumbe, es una de las más importantes en el Sur de Quito, el sector de mayor dinámica constructiva en el Distrito Metropolitano;

\subsubsection{La evaluación: antídoto contra la inercia y otros males}

Muchos programas sociales, generalmente a partir de los primeros logros, se tornan inerciales y, por consiguiente, cierran las puertas a actualizaciones y cambios necesarios que garanticen su vigencia. Si bien es cierto que en el proceso de "Solidaridad" no se advierten síntomas en esa dirección, es importante tomar en consideración el tema, tanto más si aspiramos a que el modelo sirva de base para la modernización y racionalización de las políticas de vivienda en nuestro país. En esta dirección parece necesario y oportuno ampliar los alcances de la evaluación que, actualmente, tiene como su más importante elemento, a la fiscalización técnica de la construcción del Plan de Vivienda "Quitumbe";

La evaluación considerada como parte orgánica de la opción, será herramienta imprescindible para potenciar los logros obtenidos y traducidos, principalmente, en lo que hemos aprendido. En efecto, es evidente que la opción de producción social de la vivienda, aplicada exitosamente en el Plan de Vivienda "Quitumbe", ha permitido acumular experiencia, simultáneamente con la ejecución de cada una de las cinco etapas desarrolladas desde el año 2002. El importante capital que representa la experiencia acumulada, está distribuido en los campos social, institucional y técnico. Los componentes del programa de vivienda que mayormente se han enriquecido con la experiencia acumulada son: organización y desarrollo comunitario; planificación, ejecución y administración de la construcción; organización y gestión microempresarial. No cabe duda que también se ha producido un importante enriquecimiento en la visión y alcances de la fiscalización tradicional, puesto que ésta ha debido adaptarse a la nueva forma de organización de la construcción, que involucra la producción social de la vivienda. 
Adicionalmente es necesario considerar que las actividades que forman parte de la producción social de la vivienda, al igual que toda actividad humana, no son estáticas y son perfectibles, más aún si se refieren a procesos involucrados en la dinámica del desarrollo social, como efectivamente son los que tienen lugar en el Plan de Vivienda "Quitumbe". En particular, para quienes, como parte de la carrera de Ingeniería Civil de la Universidad Politécnica Salesiana, estamos involucrados en el programa, siempre habrá mucho que hacer para mejorar.

En consecuencia, a más de la ampliación substancial del alcance de la evaluación, resulta imprescindible incluirla como componente orgánico de la producción social de la vivienda. Entre los resultados a los que podremos llegar al introducir esta práctica, tenemos los siguientes:

- Sistematización de la experiencia acumulada. Por ejemplo, actualizando, en el área técnica, las especificaciones de construcción, como base, no sólo para las siguientes etapas, sino para aplicaciones del modelo en otros escenarios;

- Cuantificación del uso del tiempo y de los materiales, en dirección a proponer acciones que permitan, por una parte, reducir los tiempos de construcción y, por otra, minimizar el desperdicio de materiales de construcción;

- Determinación de la incidencia de la mano de obra en el costo de construcción;

- Determinación de los efectos del proceso constructivo, en la calidad de la mano de obra, particularmente en relación a su capacitación y especialización;

- Valoración, en términos presupuestarios, del aporte de la comunidad en la producción social de la vivienda;

- Estandarización de los procesos de adquisición, a fin de agilitarlos y obtener los precios más bajos del mercado;

- Estandarización los controles de calidad, particularmente de los materiales de construcción entregados en obra y de los elementos constructivos producidos en ella;

- Determinación de los presupuestos definitivos, con desglose tanto en el grupo de costos directos como en el de indirectos.

Para llegar a estos resultados y, como consecuencia, mejorar los indicadores de calidad, eficiencia, 
eficacia y efectividad, así como para potenciar el efecto de las experiencias adquiridas, validándolas en el tiempo, consideramos que importante atención merecen la evaluación ex ante y ex post en cada etapa del Programa de Vivienda "Quitumbe". La evaluación ex post nos ayudará a seguir aprendiendo de la experiencia $y$, por tanto, a formular mejor los proyectos de las siguientes etapas; por su parte, la evaluación ex ante, permitirá optimizar la vía, hacia los objetivos de la etapa, seleccionando la solución más eficiente en la utilización de los recursos y, eventualmente, superando las deficiencias detectadas en el proceso de preinversión.

Sobre la evaluación hay muchas definiciones con mayor o menor retórica; una que parece clara es la siguiente: "La evaluación es una actividad que tiene por objeto maximizar la eficacia de los programas en la obtención de sus fines y la eficiencia en la asignación de recursos para la consecución de los mismos". No cabe duda que insumo importante para la evaluación, particularmente para la ex post, deben ser los resultados del seguimiento de la ejecución de obras, traducidos en los informes de fiscalización. La evaluación debe caracterizarse por la objetividad, disponibilidad 'de información suficiente y por la aplicación de métodos validos, para llegar a conclusiones y recomendaciones confiables.

\subsubsection{Evaluación ex ante}

Permitirá disponer de elementos de juicio suficientemente convincentes para la importante decisión conceptual que responda a la pregunta: ¿debe o no ejecutarse la etapa (o algún componente de ella), bajo las condiciones establecidas en la preinversión?

En principio, para la evaluación ex ante son aplicables tanto el método de "Análisis costo-beneficio" (ACB), como el método de "Análisis costo-efectividad" (ACE). Sin embargo para un proyecto social como el plan de "Solidaridad" el método ACE, traduce con mayor fidelidad la realidad. La evaluación ex ante, por tratarse de una evaluación prospectiva, permitirá valorar las potencialidades de cada etapa, antes de su ejecución y tomar los correctivos que sean necesarios.

La evaluación ex ante, en el método ACE, tendrá como premisa, la condición de que la etapa o proyecto permitirá lograr los fines que interesan a la sociedad en su conjunto, a través de la opción más eficiente. Eso implicará comparar los costos con los productos de la etapa, que en este caso serán las viviendas, los servicios 
comunitarios, la organización comunitaria y la recuperación-protección ambiental.

En la cuantificación de costos, será necesario incluir todos los recursos que la etapa requerirá durante la vida útil o sea la inversión para la ejecución y los costos de operación. La inversión para la ejecución deberá incluir los costos del terreno, de la infraestructura para vivienda, instalaciones y servicios, así el monto que represente la participación de la comunidad organizada, en la ejecución de las obras.

En relación a los productos de la etapa, habrá que precisar la demanda a ser satisfecha, quiénes van a ser los beneficiarios y su número; así como los beneficios que recibirán.

Los costos obtenidos, referidos a la unidad habitacional y sus servicios, constituirán elemento de juicio objetivo en la evaluación de las ventajas comparativas de la alternativa seleccionada para la etapa.

La evaluación ex ante, que, de ninguna manera debe llegar hasta extremos propios de las burocracias anquilosadas e ineficientes, razonablemente podrá incluir la consideración de los siguientes aspectos:

a) Identificación del problema que se resolvería con la construcción de la etapa planteada. Se refiere a la comparación entre la situación actual y la políticamente deseable. En este punto se determinara: i) las necesidades que serán satisfechas; ii) quienes integran el grupo meta de la etapa; iii) la localización del grupo-meta; iv) descripción de las alternativas consideradas para la ejecución de la etapa; v) disponibilidad de recursos;

b) Diagnóstico de la situación. Incluirá: i) descripción de la solución propuesta, así como de la expectativa que el grupo meta tiene respecto a dicha propuesta; ii) cuantificación de la cobertura existente y la deseable, así como de los estándares de calidad previstos para la etapa;

c) Resumen ejecutivo sobre el análisis y comparación de alternativas para la selección de la mejor;

d) Análisis de la alternativa seleccionada, inclusive de: i) memoria técnica; ii) planos y diseños; iii) cronograma y presupuesto; iv) especificaciones técnicas;

e) Análisis del organigrama para la ejecución de la etapa;

f) Alcance y normas para la fiscalización; 
g) Reglamento de adquisiciones y contrataciones;

h) Identificación de los servicios y provisiones a cargo de las microempresas de "Solidaridad".

\subsubsection{Evaluación ex post}

La evaluación ex post estará constituida de: i) evaluación de procesos; y, ii) evaluación de impacto.

a) La evaluación de procesos (procesos constructivos, procesos de adquisiciones, proceso de administración de la construcción, procesos de producción de materiales de construcción y otros servicios en las cooperativas de "Solidaridad", proceso de seguimiento y fiscalización de la construcción, procesos de control de calidad), será una evaluación continua y permitirá ir determinando el nivel en el que, los componentes de la etapa, vayan contribuyendo a la consecución de sus fines. En realidad se tratará de una evaluación para corregir oportunamente los desfases que se produzcan en la ejecución de la etapa. Se dice que la evaluación de procesos mira hacia adelante, en tanto en cuanto tiende a correcciones y readecuaciones oportunas. En consecuencia, esta evaluación permitirá disminuir los niveles de incertidumbre en las decisiones cotidianas, particularmente en las relacionadas con los procesos constructivos. Con ciertos correctivos que seamos capaces de introducir en las acciones de fiscalización, ésta debe representar buena parte de la evaluación de procesos;

b) La evaluación de impacto determinará la medida en la que sean alcanzados los objetivos de la etapa. Esta evaluación resultará de fundamental importancia, para "'exportar" la opción "Solidaridad" y, por tanto, sus objetivos están más allá de "Quitumbe" y "Solidaridad": deben estar en las instancias llamadas a racionalizar las políticas de vivienda en el Ecuador.

\subsubsection{Responsables de la evaluación}

Tanto la evaluación ex ante como la evaluación ex post, del Programa de Vivienda "Quitumbe", en primera instancia, deberían ser internas, pero además integrales y participativas. La ventaja de la evaluación interna, radicará en permitir a los actores del proyecto comprender mejor las activida- 
des que se evalúan y, en consecuencia, participar con mayores y mejores bases en la ejecución de la etapa en curso y de las siguientes. Al hacer la evaluación participativa, se armonizarán los principios del proceso evaluativo, con los de autogestión, solidaridad y responsabilidad comparti$\mathrm{da}$, inherentes al modelo de producción social de la vivienda que pone en práctica "Solidaridad". El carácter integral se refiere a que en la evaluación intervendrán los actores de todos los componentes de la etapa: planificadores y diseñadores, constructores, fiscalizadores, proveedores internos, administradores $y$, por cierto, el grupo meta de la etapa evaluada; de esta manera, la evaluación interna será, en realidad, evaluación externa para cada componente de ella.

No se descarta que en la evaluación interna surjan discrepancias importantes entre sus actores. En este caso, probablemente, será conveniente optar por la evaluación externa.

\section{Racionalidad, creatividad y transparencia: condiciones para la viabilidad financiera}

Los problemas cotidianos del desarrollo evidencian que son escasas las posibilidades de concretar la viabilidad financiera de los proyectos sociales de desarrollo, en circunstancias en las que, las demandas sociales, adecuadamente diseñadas, aumentan y donde las probabilidades de éxito, de las demandas formuladas sin racionalidad, son nulas. Han sido frecuentes y patéticos en nuestro país los casos de no utilización o subutilización de recursos financieros disponibles, inclusive los de asistencia externa no reembolsable, debido, principalmente, a que los proyectos han estado lejos de la racionalidad en su formulación. Estos son los surgidos de la improvisación, del cositerismo y clientelismo políticos. Pero también hemos podido constatar como, proyectos sociales, hasta cierto punto utópicos por la magnitud de la demanda financiera, finalmente han sido ejecutados, gracias a que, la racionalidad en la formulación, ha facilitado su elegibilidad para programas de asistencia de doble vía, es decir, aquéllos en los que, por una vía fluyen los recursos financieros $y$, por la otra, el aporte de la comunidad al desarrollo social, a la protección y recuperación de los recursos renovables, en fin, a la generación de nuevos modelos de desarrollo integral y digno. 
La racionalidad en la formulación de los proyectos sociales de desarrollo involucra:

a) Obedecer a una planificación seria y transparente;

b) Haber culminado un proceso de preinversión, que incluya la factibilidad integral de la propuesta;

c) Evidenciar eficiencia en la utilización de los recursos;

d) Demostrar la eficacia con la que se logran los objetivos;

e) Evidenciar la equidad de la propuesta;

f) Evidenciar la correspondencia entre la organización de la comunidad y los retos del proyecto, en las etapas de inversión y operación.

Estas condiciones cumple el Programa de Vivienda "Quitumbe", como se desprende de lo indicado en el punto 3.2.1. Por tanto, se trata de un programa elegible en la competencia, cada vez más difícil, por la captación de recursos financieros, siempre deficitarios para la ejecución de las siguientes etapas. En esta dirección, no cabe duda que la evaluación orgánica propuesta líneas arriba tendrá importante incidencia positiva.

\section{La Universidad en "Qui- tumbe" y una potencia preo- cupante}

La Carrera de Ingeniería Civil de la Universidd Politécnica Salesiana, consideró su participación en el Programa de Vivienda "Quitumbe", como parte de una cooperación mutua $y$, en esa dimensión hemos desarrollado nuestro trabajo desde el año 2002. Estamos terminando la fiscalización de la quinta etapa del Programa. Nuestras acciones, caracterizadas, en las primeras etapas, por la rigurosidad y frialdad de la lógica ingenieril, finalmente, han aterrizado en un nuevo modelo de fiscalización, adaptado por la fuerza del sentido común, a la opción de producción social de la vivienda. El nuevo modelo de fiscalización se caracteriza por garantizar la calidad de trabajos y obras, sobre la base, principalmente, de la participación cada vez más comprometida, de nuestros ingenieros, arquitectos y estudiantes; en conclusión podríamos definirlo en pocas palabras: COLABORACIÓN PARA LA PREVENCIÓN.

Probablemente, en nuestras condiciones, es difícil asimilar esta forma de fiscalización, debido a la inercia de la tradición y, sobre todo, a las 
desagradables experiencias de la fiscalización en el país, particularmente en la obra pública. Las dudas y preocupaciones pierden piso frente a dos condiciones sencillas que debe cumplir la fiscalización: i) transparencia en la selección de fiscalizadores; ii) transparencia en el trabajo de los fiscalizadores; es decir, interdependencia de dos condiciones, en la que, la segunda es razón y consecuencia de la primera. No cabe duda que el "Sistema Preventivo" tiene proyecciones y aplicaciones inmensas e imprevisibles.

$\mathrm{Al}$ ser esta participación un caso de cooperación de la universidad para el desarrollo, resulta oportuno considerarla en el contexto de una importante presentación, hecha en el Primer Congreso Internacional "Universidad, Desarrollo y Cooperación", cumplido en días pasados en la ciudad de Cuenca. Se trata de la ponencia del doctor Enrique Ayala, quien, propone eliminar, de la agenda de vinculación con la colectividad de las universidades, la formulación y ejecución de proyectos de desarrollo. Antes de comentar la propuesta, conviene señalar los siguientes elementos de juicio:

a) La participación de las universidades y escuelas politécnicas en la preinversión de proyectos está prevista, expresamente, en el Art. 5 de la Ley de Consultoría, que estipula: “... Las universidades y escuelas politécnicas podrán también ejercer la consultoría de conformidad con las disposiciones legales o estatutarias que normen su vida jurídica";

b) La inclusión de esta disposición legal se consideró, en su tiempo, una conquista de la Universidad ecuatoriana, propuesta y respaldada en el H. Congreso Nacional, por legisladores vinculados con la izquierda política;

c) En nuestro país, en varios procesos de preinversión de proyectos de desarrollo, han participado, cumpliendo las normas legales y las bases de licitación específicas, universidades europeas y americanas. Como ejemplo hacemos referencia a casos puntuales que, en nuestro ejercicio profesional, conocimos directamente:

- La Universidad Técnica de Braunschweig (Alemania), participó en los estudios de factibilidad del proyecto hidroeléctrico Paute-Mazar; a su cargo estuvo el estudio del modelo hidráulico para la alternativa con presa de 
gravedad. El financiamiento provino de una operación reembolsable del BID;

- Las universidades de Utah, Arizona, Washington y California, participaron en el programa de reestructuración del régimen institucional del agua en Ecuador. El financiamiento vino de una operación reembolsable del BID;

- Los modelos hidráulicos, de la presa "Agoyán" y del aliviadero de excedentes de la presa "Daule - Peripa", también fueron investigados en universidades europeas. Los costos, igualmente, fueron los de mercado.

Las razones del doctor Enrique Ayala, probablemente son justificadas y compartimos su preocupación, en tanto, durante los últimos quince años se han advertido abusos en la utilización de la facultad prevista en el mencionado Art. 5 de la Ley de Consultoría; sin embargo, de ser este el problema, debemos admitir que en nuestro país es común que las disposiciones legales permisivas y restrictivas terminen convirtiéndose en fuente de inmoralidad; por tanto, la solución no está en derogar dichas normas.

De otra parte, la eliminación de la facultad a las universidades de participar en preinversión de proyectos tendría signo negativo, entre las variables de las que depende la calidad profesional, particularmente, de quienes egresan de las carreras de ingeniería.

Sobre la solución al problema que habría dado origen a la ponencia del doctor Enrique Ayala, es oportuno meditar. Por ejemplo, podría resultar conveniente establecer, en el reglamento a la Ley mencionada, elementos y condiciones de transparencia; también puede ser útil asegurar, con mecanismos a definirse, que la participación de las universidades en proyectos de desarrollo, se realice en el marco de cooperaciones de doble dirección y sin fines de lucro.

\section{Algunas conclusiones}

6.1 Las políticas para la vivienda de interés social no deben ser ni improvisadas, ni imperativas, sino caracterizadas por la racionalidad, el enfoque integral y la previsión de mecanismos de coordinación y concertación con los sectores involucrados; 
6.2 Para el desarrollo de programas de vivienda de interés social, debería privilegiarse la opción de producción social de la vivienda, por ejemplo, sobre la base de la experiencia validada, única en Ecuador, de la Asociación de Cooperativas "Solidaridad";

6.3 El modelo de producción social de la vivienda debe incluir, como proceso orgánico, las evaluaciones ex ante y ex post, concebidas como herramientas para maximizar la eficacia en la obtención de los objetivos y la eficiencia en el uso de recursos;

6.4 La inclusión, en los programas de vivienda de interés social, de componentes como: recuperación de los recursos renovables, desarrollo social, desarrollo urbano, desarrollo microempresarial, etc., conjuntamente con las premisas de eficacia y eficiencia, referidas en el punto precedente, generarán condiciones de selectividad, para asis- tencias no reembolsables o financiamiento blando;

6.5 La participación de la universidad, en los programas de interés social, al igual que en cualquier programa de desarrollo, debería ser enfocada como contraparte técnica de cooperaciones con doble vía.

\section{Bibliografía}
"DECLARACIÓN DE GUADALUPE"
2005 X Foro Iberoamericano de Ministros del Sector de la Vivienda y Desarrollo Urba- no. Guadalupe, septiembre.
BAYAS, Omar; CALERO, Iván; PAZ, Jorge
2006 "Informe de Evaluación del Programa de Vivienda Po- pular Primavera I y II, cons- truido en la ciudad de Ma- chala". Universidad Politéc- nica Salesiana, diciembre.

COHEN, Ernesto; FRANCO, Rolando

2003 "Evaluación de Proyectos Sociales". Banco Interamericano de Desarrollo.

Ley de Consultoría

2004 Codificación 24. Registro Oficial 455 de 5 de noviembre. 